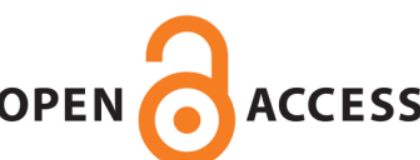

\title{
Application of 3-D FKK filtering in 3-D high-density onshore seismic field data
}

Liao, Jianping; Fu, Songyuan; Xu, Yungui; Li, Weibo; Chen, Jianxiong; Wang, Huazhong; Peng, Suping; Hursthouse, Andrew

Published in:

Journal of Environmental \& Engineering Geophysics

DOI:

10.2113/JEEG23.3.369

Published: 01/09/2018

Document Version

Peer reviewed version

Link to publication on the UWS Academic Portal

Citation for published version (APA):

Liao, J., Fu, S., Xu, Y., Li, W., Chen, J., Wang, H., Peng, S., \& Hursthouse, A. (2018). Application of 3-D FKK filtering in 3-D high-density onshore seismic field data. Journal of Environmental \& Engineering Geophysics, 23(3), 369-376. https://doi.org/10.2113/JEEG23.3.369

\section{General rights}

Copyright and moral rights for the publications made accessible in the UWS Academic Portal are retained by the authors and/or other copyright owners and it is a condition of accessing publications that users recognise and abide by the legal requirements associated with these rights. 
Use of this publication is subject to SEG (Society of Exploration Geophysicists) terms of use and conditions.

For more information: https://seg.org/Publications/Policies-and-Permissions/Open-Access-Policy 


\title{
Application of 3-D FKK Filtering in 3-D High-density Onshore Seismic Field Data
}

Jianping Liao ${ }^{1,2}$, Yungui $\mathrm{Xu}^{3 *}$, Hexiu $\mathrm{Liu}^{1}$,Weibo $\mathrm{Li}^{4}$, Jianxiong $\mathrm{Chen}^{5}$, Huazhong Wang ${ }^{6}$, Suping Peng ${ }^{2}$, Andrew Hursthouse ${ }^{1,7}$

${ }^{1}$ Hunan Provincial Key Laboratory of Shale Gas Resource Utilization, Hunan University of Science and Technology, Xiangtan,411201, China. Email: 1020116@hnust.edu.cn

${ }^{2}$ State Key Laboratory of Coal Resources and Safe Mining, China University of Mining \& Technology, Beijing,100083, China

${ }^{3}$ School of Geoscience and Technology, Southwest Petroleum University, Chengdu, 610500,China. E-mail: yungui.xu@gmail.com

${ }^{4}$ BGP, CNPC, Zhuozhou, 072751, China

${ }^{5}$ Department of Earth Science, Rice University, Houston, TX 77005. USA

Email: Jianxiong.chen.rice@gmail.com

${ }^{6}$ WPI, School of Ocean and Earth Science, Tongji University, Shanghai, 200092, China

${ }^{7}$ School of Science \& Sport, University of the West of Scotland, Paisley PA1 2BE, UK

\begin{abstract}
For linear noise such as seismic ground roll, 3-D frequency-wavenumber-wavenumber (3-D FKK) domain filtering suppression is better than 2-D frequency-wavenumber (F-K) domain filtering. In recent years, with the continuous development of computer processing speed and memory capacity, high-density data acquisition in seismic exploration has been widely applied in the hydrocarbon industry, opening up the application of 3-D FKK filtering methods. We applied the 3-D FKK filtering software developed locally by ourselves to a 3-D high-density onshore seismic field dataset from a coal mines in western China. The case study demonstrates that the linearity of the noise in the field data is better represented by constructing the single shot records as a minimum dataset. Both theoretical synthetic models and the 3-D high-density onshore seismic field data numerical filtering experiments demonstrate that the feasibility of our 3-D FKK filtering software. The filtering effects are good.
\end{abstract}

\section{Introduction}

Currently, 3-D seismic exploration is a popular technique (e.g., Yan et al., 2017), but the 3-D filtering has not developed significantly. The common approach is to first filter data inline and then crossline (two-pass filtering method, Stewart, 1989; Peardon and Bacon, 1992; Li and Larner, 1993) to replace the true 3-D filtering, which is low efficiency because of switching data between the horizontal and vertical directions.

Separating linear coherent noise, such as ground roll from reflections, remains a key challenge in seismic processing. The coherent noise, especially the ground roll, are difficult to grasp in the form of 3-D shot gather datasets in 2-D. The apparent velocity of the coherent noise identified in 2-D is different from its true apparent velocity, and the apparent velocity of each shot gather is also different. Therefore, it is not ideal to filter out 
the coherent noise with a two-pass filtering method.

The distribution of signal and ground roll are different in the frequency-wavenumber domain, so we can identify the noise energy in frequency-wavenumber relationship, and we can design the factors removed, we cut off the energy that is higher (or lower) than a certain dip angle to allow for filtering. The specific implementation of the cut-off can be carried out in the time-space domain (Treitel et al., 1967; Hale and Claerbout, 1983; Claerbout, 1985; Li and Larner, 1993; Liao et al., 2018); or in the frequency-wavenumber domain (Wiggins, 1966; Peardon and Bacon, 1992). As seismic studies undertake investigation of more complex geology, more innovative methods were needed. Examples include derivative filters (Melo et al., 2009; Nie et al., 2014), empirical model decomposition (Bekara and van der Baan, 2009), time-frequency transforms (Askari and Siahkoohi, 2008; Liu and Fomel, 2013), and compressed sensing and sparsity promotion (Wang et al., 2008; Gholami, 2014). A generic shortcoming of these methods is the operational loss of the lower end of the body wave frequency spectrum. By adapting the redundant lifting scheme, a wavelet transform method, to seismic data, Aghayan et al., (2016) determined how the wavelet domain can be used to suppress coherent and random noise. They proposed the new method that was wavelet-based ground roll noise suppression using a synthetic shot gather and two real gathers.

High-density seismic exploration has been developed in recent years in order to improve the quality of seismic data acquisition and processing techniques, which use a single-sensor recording, vibroseis alternating scanning and digital detector technologies (Rajab et al., 2006). High-density seismic exploration data with small temporal and spatial sampling intervals, and high folding numbers, eliminates the influence of the false frequency in processing, which improves the separation of the signal and noise in the 3-D FKK domain greatly.

With different ways to realize filtering the two-pass filtering method first filters in the FKx domain and then in the FKy domain. While one-pass filtering method removes the ground roll energy in the FKK domain directly. Peardon and Bacon (1992) used a marine seismic section that was a single line from a 3-D survey for examples of 3-D FKK filtering application. In this paper, we extended their research and applied 3-D FKK filtering method (Stewart, 1989) on a 3-D high-density onshore seismic field data from a coal mines in western China, which was typically noisier than marine seismic field data. Both theoretical synthetic models and the 3-D high-density onshore seismic field data numerical filtering experiments demonstrate that the feasibility of our 3-D FKK filtering software. The filtering results show that the denoising effects are good.

\section{Methodology}

In the frequency domain, if we assume that the critical apparent velocity of the noise is $V_{L}$, all the energy that is lower than this apparent velocity is regarded as noise, our 3-D one-pass FKK filtering cut-off factor $F\left(\omega, k_{x}, k_{y}\right)$ can be made as follow:

$$
F\left(\omega, k_{x}, k_{y}\right)= \begin{cases}1 & \omega / \sqrt{k_{x}^{2}+k_{y}^{2}} \geq V_{L} \\ 0 & \omega / \sqrt{k_{x}^{2}+k_{y}^{2}}<V_{L}\end{cases}
$$

As for the 3-D two-pass FKK filtering, our cut-off factor $F^{\prime}\left(\omega, k_{x}, k_{y}\right)$ can be made as follow: 


$$
F^{\prime}\left(\omega, k_{x}, k_{y}\right)=F^{\prime \prime}\left(\omega, k_{x}\right) g F^{\prime \prime}\left(\omega, k_{y}\right)
$$

Where

$$
\begin{aligned}
& F^{\prime \prime}\left(\omega, k_{x}\right)= \begin{cases}1 & \omega / k_{x} \geq V_{L} \\
0 & \omega / k_{x}<V_{L}\end{cases} \\
& F^{\prime \prime}\left(\omega, k_{y}\right)= \begin{cases}1 & \omega / k_{y} \geq V_{L} \\
0 & \omega / k_{y}<V_{L}\end{cases}
\end{aligned}
$$

It is found that the 3-D two-pass FKK filtering is a tetrahedral shape, while 3-D one-pass FKK filtering is a cone, so there are some distinct differences between them. Here, we refer the readers to (Stewart, 1989; Peardon and Bacon, 1992).

\section{Synthetic Data Example}

In (Peardon and Bacon, 1992), they have demonstrated that 3-D FKK is better than 2-D FK filtering with synthetic data. In this paper, we synthesized a theoretical record and used it to verify the efforts of our 3-D FKK filtering software. The wavelet in the synthetic record was defined as following:

$$
\omega=\frac{20}{t} e^{-2000 t^{2}} \sin (30 \pi t) \cos (62.5 \pi t)
$$

Where, the dominant frequency of the wavelet was $25 \mathrm{~Hz}$.

The geometry was as following: The detector arrangement was arranged in single line, 100 traces for each source, the trace interval was $5 \mathrm{~m}$, and the time sampling interval was $2 \mathrm{~ms}$. The source point was perpendicular to the receiver line and the shot interval was also $5 \mathrm{~m}$.

In the Fig.1, the direct arrival wave velocity was $1,000 \mathrm{~m} / \mathrm{s}$, the reflection wave velocity was $1,500 \mathrm{~m} / \mathrm{s}$, the ground roll velocity was $500 \mathrm{~m} / \mathrm{s}$. In the furthest source point, the noise that should be linearly distributed was shown as an arc in the section, which proved our understanding that linear noise was non-linear in the two-dimensional body. In order to eliminate linear noise well, the filtering method should be carried out in three dimensions.

It can be seen from Fig.1 that 3-D FKK filtering method was excellent for removing linear noise. The linear noise was completely suppressed, and the signal was preserved very well. The signal and noise in the frequency spectrum analysis of Fig.1 were well separated, which fully demonstrated the good denoising effects of 3-D FKK filtering on synthetic data.

\section{3-D FKK Filtering Experiments on 3-D High-density Onshore Seismic Field Data}

In (Peardon and Bacon, 1992), they used a marine seismic section that was a single line from a 3-D survey for examples of filtering application. We extended their work and applied 3-D FKK filtering to an onshore seismic field data, which was typically noisier than marine seismic field data. The 3-D high-density onshore seismic field dataset was acquired from a coal mines region in western China, where strong coherent surface-generated noise was prevalent. Because the field dataset was large and in order to demonstrate our 3-D FKK filter effectively, we followed Peardon and Bacon (1992) in which we extracted a shot line and a 
receivers line perpendicular with each other from the 3-D field data forming a cross-shaped geometry. As a result, the extracted three-dimensional data body had only one folding.

The geometry of the extracted onshore seismic field data was as follows: there were 158 shots, each shot had 160 traces with single line receiving, the trace spacing was $10 \mathrm{~m}$. The shot interval was $10 \mathrm{~m}$. There were 25,280 traces in total, each trace had 2,001 time sample points with a time sampling interval of $1 \mathrm{~ms}$. The total time length was 2 seconds in each trace. A cross arrangement was used, the minimum offset was $5 \mathrm{~m}$, the longest distance between the source and the receiver was $795 \mathrm{~m}$.

Figures 2-4 showed three typical seismic shot gathers of these field data. It could be seen that the ground roll energy was quite prominent. The filtered shot gathers were shown in Fig.2(b), Fig.3(b) and Fig.4(b) respectively. Fig.2(a) was the near source point's arrange single shot raw records; Fig.2(b) was single shot records after 3-D FKK filtering in inline direction; Fig.2(c) was the filtered noise. Fig.3(a) was the intermediate source point's arrange single shot raw records; Fig.3(b) was single shot records after 3-D FKK filtering in inline direction; Fig.3(c) was the filtered noise. Fig.4(a) was the far source point's arrange single shot raw records; Fig.4(b) was single shot records after 3-D FKK filtering in inline direction; Fig.4(c) was the filtered noise.

The main objects of Figs.2, 3 and 4 were to eliminate the ground roll. The wavefield filtered by Fig.2 were ground roll, but the interference wavefield with cross skew were left in the effective wavefield from the zero offset (the energy of this part was not completely eliminated, it may be related to the data acquired by the cross 3-D onshore survey). In view of the noise filtered from Fig.3(c), a small percentage of shallow-layer direct arrival wave and shallow reflection wave at the top of Fig.3 were also cut off. In Fig.4, effective waves were also degraded from the far shot and receiver distance at $800 \mathrm{~ms}$. Although the ground roll was not as prominent as that of Fig.2, but the elimination effect was also good for Fig.4.

Figure 2 filtering effect was the best, without losing any effective wave, the noise was almost totally removed without impairing the quality of the underlying seismic field data. The ground roll was completely filtered. Fig.3 was the second best. Fig.4 was less successful compared to Fig.2 and Fig.3. It could be found out that all these three typical shot gathers showed good level of denoising. The signals were preserved relatively well.

Figure 5(a) was a time slice at $1500 \mathrm{~ms}$ of a stack profile with 1 fold before 3-D FKK filtering using 158 shots, Fig.5(b) was a time slice at $1500 \mathrm{~ms}$ of a stack profile with 1 fold after 3-D FKK filtering using 158 shots. The ground roll shape was circle, which were corresponding to and consistent with Peardon and Bacon (1992) paper's Fig.18(c) and Fig.19(c). This could demonstrate that the 3-D FKK software we developed was correct. It could also be shown that the signal-to-noise ratio of Fig.5(b) was much better than that of Fig.5(a).

Figure 6(a) showed a typical shot gather after a conventional 2-D FK filtering, noting that residual noise was still strongly evident in the section. Fig.6(b) was the same typical shot gather after 3-D FKK filtering in inline direction. The 3-D FKK filtering showed a significant improvement, the low-frequency digital intersection of the ground roll were removed by 3-D FKK filtering, the noise were completely suppressed by 3-D FKK filtering. The improvement effects of 3-D FKK filtering were relatively significant, and they were much better compared to 2-D FK filtering.

\section{Conclusions}


We applied one-pass 3-D FKK filtering software to a 3-D high-density onshore seismic field data from a coal mines in western China to suppress the linear noise and the ground roll in the 3-D. Denoising effects of the near source point, intermediate source point and far source point's typical shot gathers were good. The signals were all preserved well. The improvements of denoising effects of 3-D FKK filtering were much better compared to 2-D FK filtering.

\section{Discussion}

Because the 3-D FKK filtering method requires the 3-D data to be transferred into computer memory modules to undertake the 3-D Fourier transform, consumption of computer memory is very high. In addition, the processing of data truncation, the FFT (fast Fourier transform) and application of the cut-off factors can affect the final denoising effects.

Although its computational efficiency has been improved, it is believed that with the continuous improvements in computational power and continuous improvements of hardware speed and capacity, further improvements in data denoising processing will be achieved.

\section{Acknowledgements}

This research was financially supported by the National Natural Science Foundation of China (41274126), State Key Laboratory for Coal Resources and Safe Mining, China University of Mining \&Technology (SKLCRSM17KFA01, SKLCRSM11KFB01), open fund of Hunan Provincial Key Laboratory of Shale Gas Resource Utilization, Hunan University of Science and Technology. ASH acknowledges the support of Hunan Provincial Government and Hunan University of Science \& Technology through High End Expert Scholarship.

\section{References}

Aghayan A, Jaiswal P, Siahkoohi H R, 2016, Seismic denoising using the redundant lifting scheme: Geophysics, 81,V249-V260.

Askari R, Siahkoohi H R, 2008, Ground roll attenuation using the S and x-f-k transforms: Geophysical Prospecting, 56, 105-114.

Bekara and van der Baan, 2009, Random and coherent noise attenuation by empirical mode decomposition: Geophysics, 74, V89-V98.

Claerbout, J.F., 1985, Imaging the earth’s interior (Vol.1): Blackwell scientific publications, Oxford.

Gholami A, 2014, Non-convex compressed sensing with frequency mask for seismic data reconstruction and denoising: Geophysical Prospecting, 62,1389-1405.

Hale, D., and Claerbout, J., 1983, Butterworth dip filters: Geophysics, 48, 1033-1038.

Jianping Liao, Zhenwei Guo, Hexiu Liu, Shixin Dai, Yanlin Zhao, Lixin Wang, Huazhong Wang, Andrew Hursthouse, 2017, Application of Frequency-dependent Traveltime Tomography to 2D Crosswell Seismic Field Data: Journal of Environmental \& Engineering Geophysics, 22, 421-426.

Li, J., and Larner, K., 1993, Differential-equation-based seismic data filtering: Geophysics, 58, 1809-1819.

Jianping Liao, Hexiu Liu, Weibo Li, Zhenwei Guo, Lixin Wang, Huazhong Wang, Suping Peng, Andrew Hursthouse, 
2018, 3-D Butterworth filtering for 3-D high-density onshore seismic field data: Journal of Environmental \& Engineering Geophysics, 23,223-233.

Li W., Li P., and Wang H., 2009, Design and realization of the Butterworth filter, in 2009 SEG Annual meeting, Society of Exploration Geophysicists, 3188-3192.

Liu Y, Fomel S, 2013, Seismic data analysis using local time-frequency decomposition: Geophysical Prospecting, 61,516-525.

Melo P E M, Porsani M J, Silva M G, 2009, Ground-roll attenuation using a 2D time-derivative filter: Geophysical Prospecting, 57,343-353.

Nie, P., L. Zhang, Y. Li, B. Yang, and X. Luan, 2014, Using the Directional Derivative Trace Transform for Seismic Wavefield Separation: IEEE Transactions on Geoscience \& Remote Sensing, 52,3289-3298.

Peardon, L. G., and C. W. M. Bacon, 1992, An introduction to FKK techniques: First Break, 10, 113-123.

Rajab,M., Al-Hakim, I., Garrity, J., Gallaway, P., Westcott, R., and Perrin, T., 2006, Single-sensor recording- a case study from a mature field in the Partitioned Neutral Zone, Kingdom of Saudi Arabia and Kuwait. in 2006 SEG Annual Meeting, Society of Exploration Geophysicists,562-565.

Stewart, R.R., 1989, 3-D FK Filtering, 59th Annual Meeting and International Exposition, SEG, Expanded Abstracts, 1123-1124.

Treitel, S., Shanks, J., and Frasier, C., 1967, Some aspects of fan filtering: Geophysics, 32, 789-800.

Wang, D., R. Saab, Ö. Yilmaz, and F. Herrmann, 2008, Bayesian wavefield separation by transform-domain sparsity promotion:Geophysics, 73, A33-A38.

Wiggins, R., 1966, w- k filter design: Geophysical Prospecting, 14, 427-440.

Yan, Y., Zhu, X., Jiao, J., Deng, P., Yang, B., Fu, L., Oropeza, E., Chen, J. and Mueller, S., 2017, Permian Basin seismic data reprocessing: A case study. SEG Annual Meeting, Society of Exploration Geophysicists, 3987-3991. 

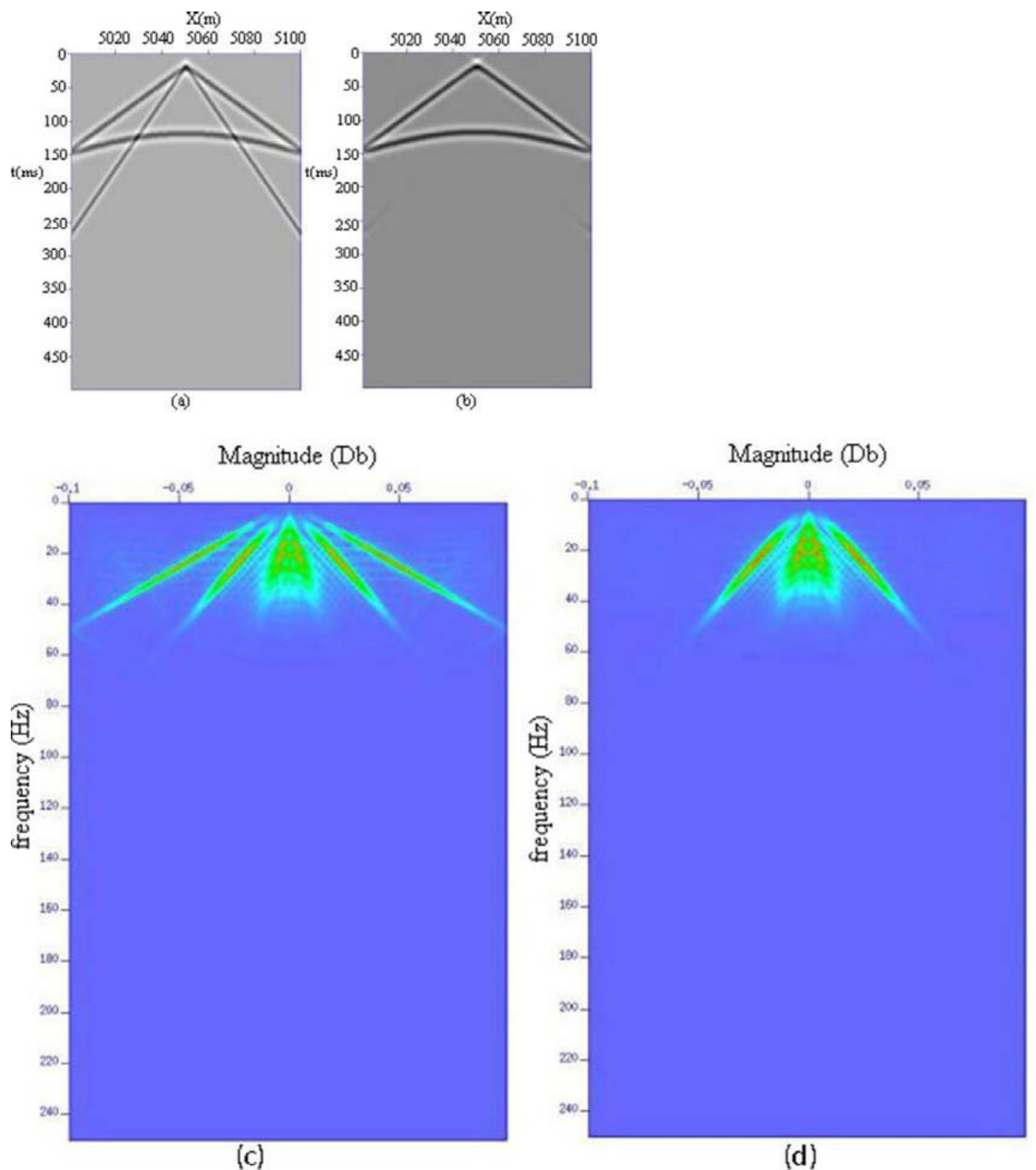

Fig. 1 (a) was near source point's arrange single shot records before filtering; (b) was near source point's arrange single shot records after filtering; (c) was single shot records' frequency spectrum before filtering; (d) was single shot records' frequency spectrum after filtering. 


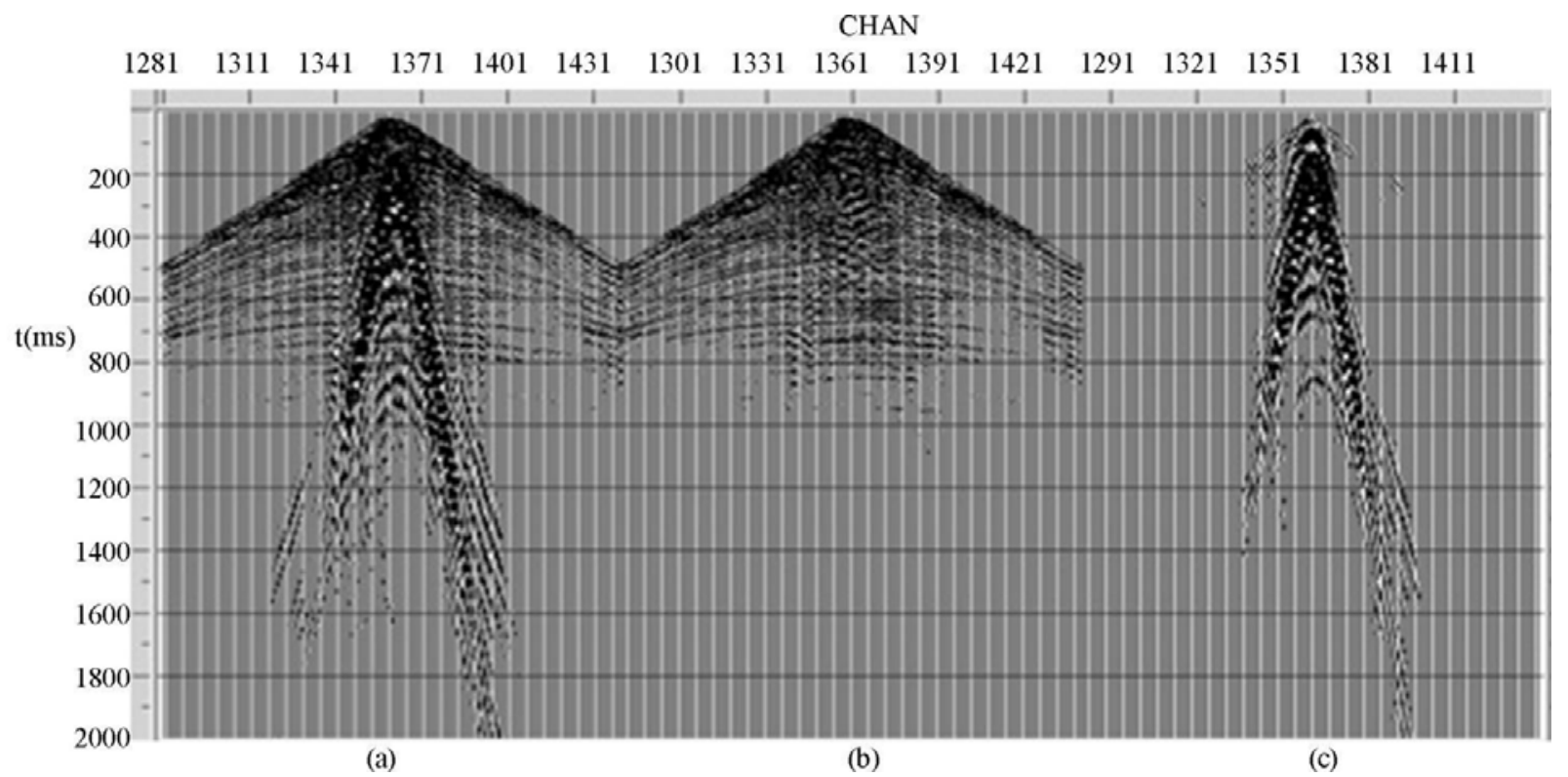

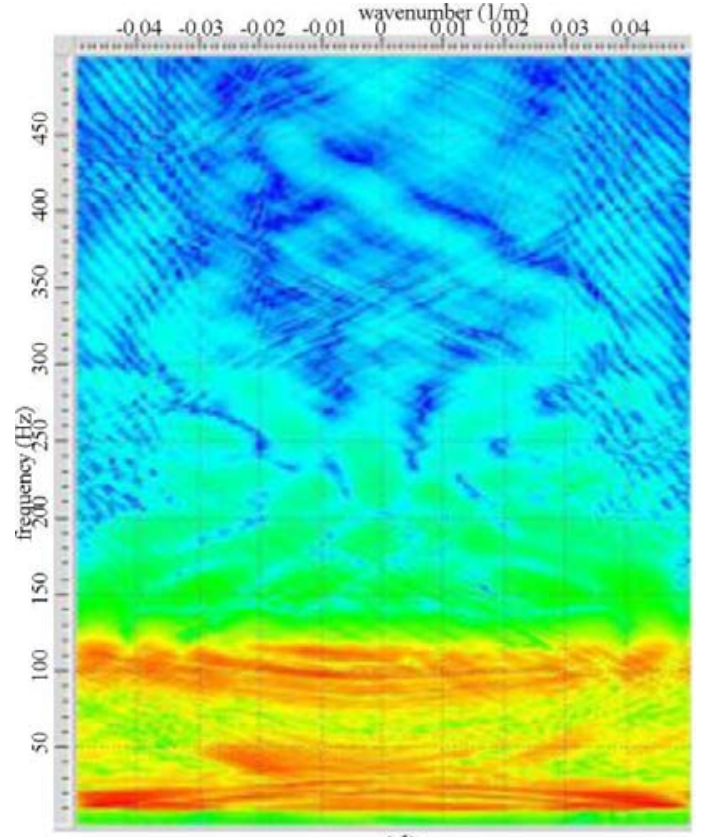

(d)

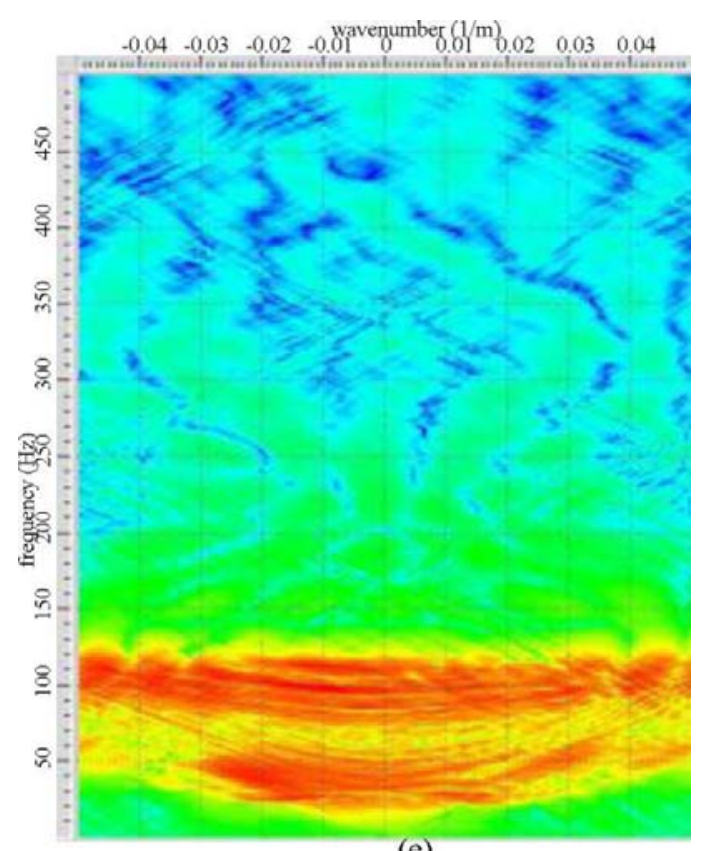

(e)

Fig. 2 (a) was near source point's arrange single shot raw records; (b) was single shot records after 3-D FKK filtering in inline direction; (c) was the filtered noise; (d) was single shot records' frequency spectrum before filtering; (e) was single shot records' frequency spectrum after filtering 


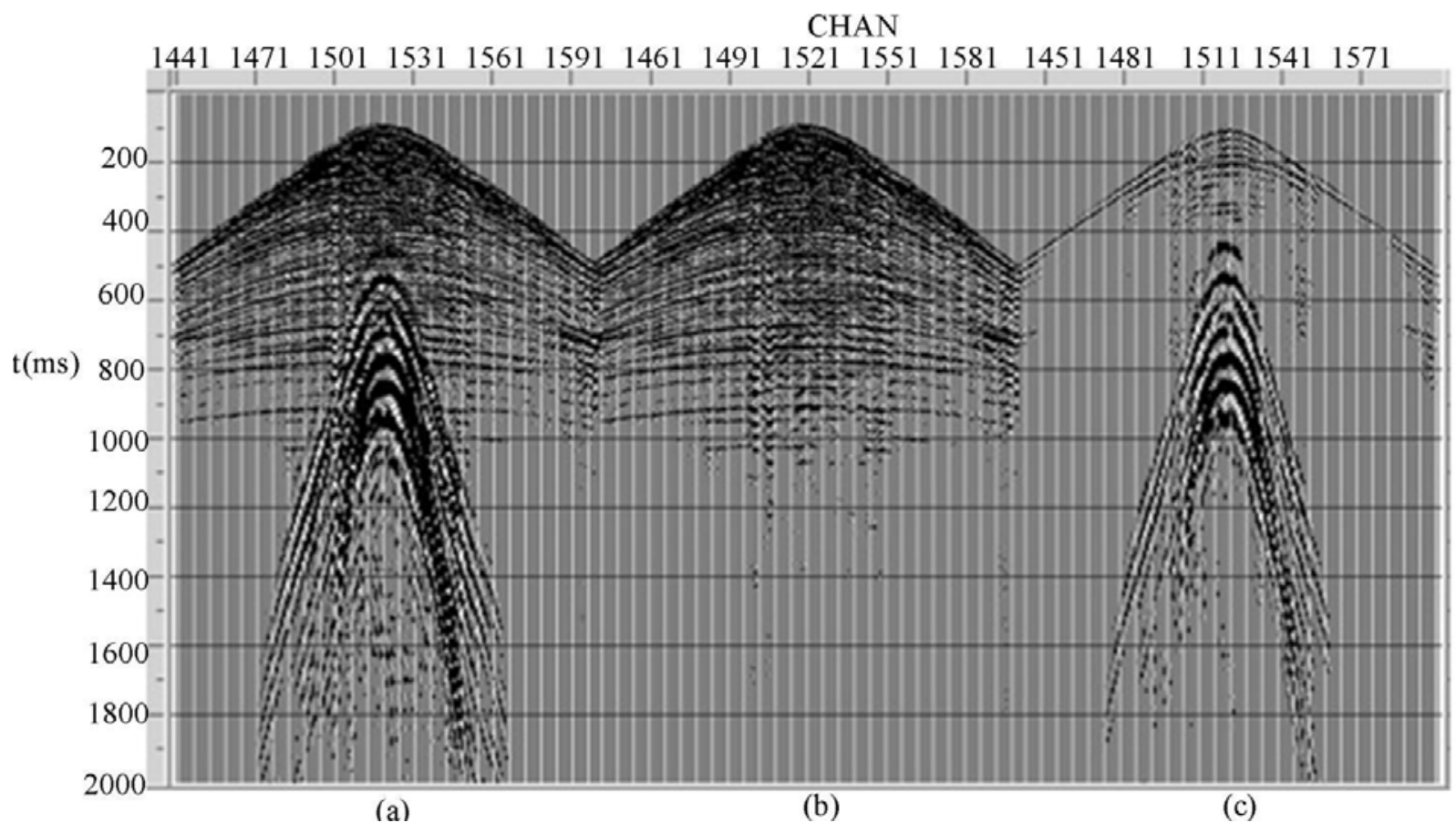

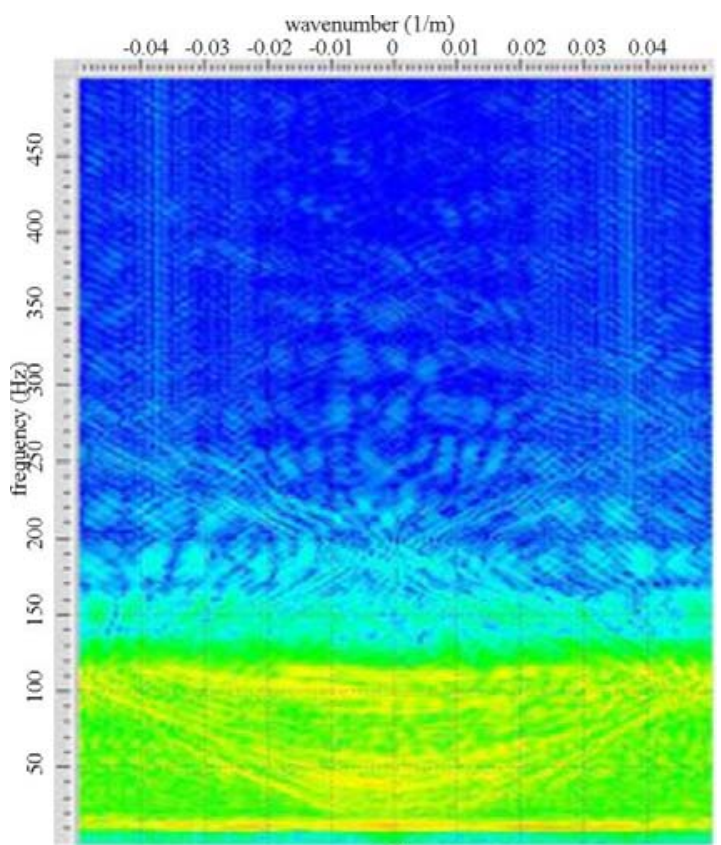

(d)

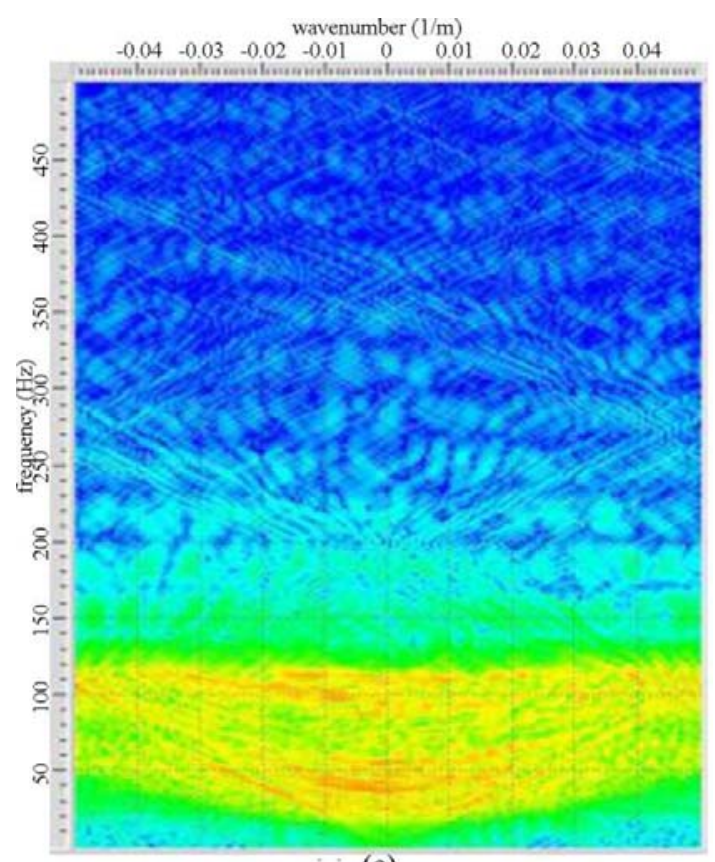

(e)

Fig. 3 (a) was mediate source point's arrange single shot raw records; (b) was single shot records after 3-D FKK filtering in inline direction; (c) was the filtered noise; (d) was single shot records' frequency spectrum before filtering; (e) was single shot records' frequency spectrum after filtering 


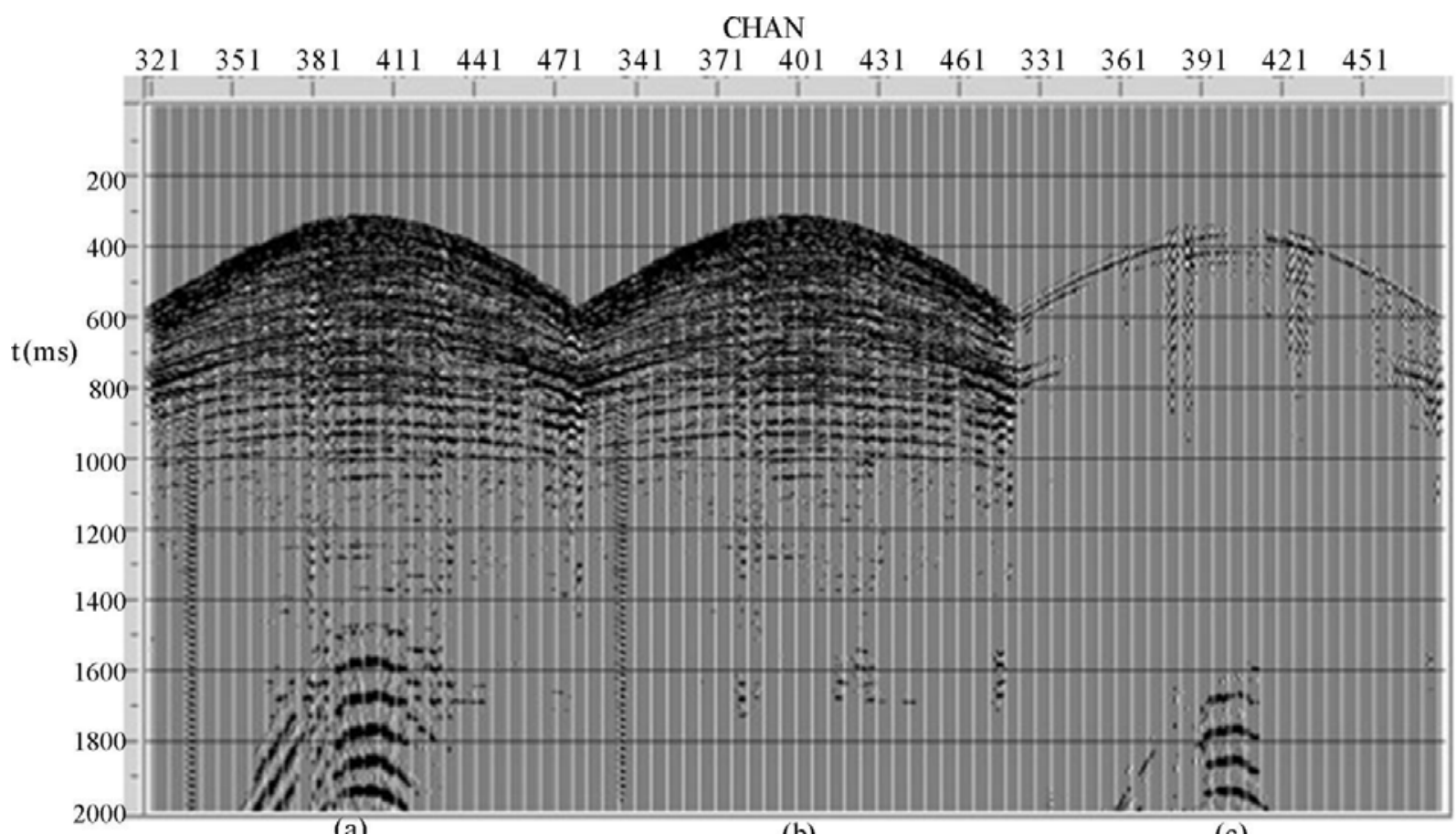

(a)

(b)

(c)
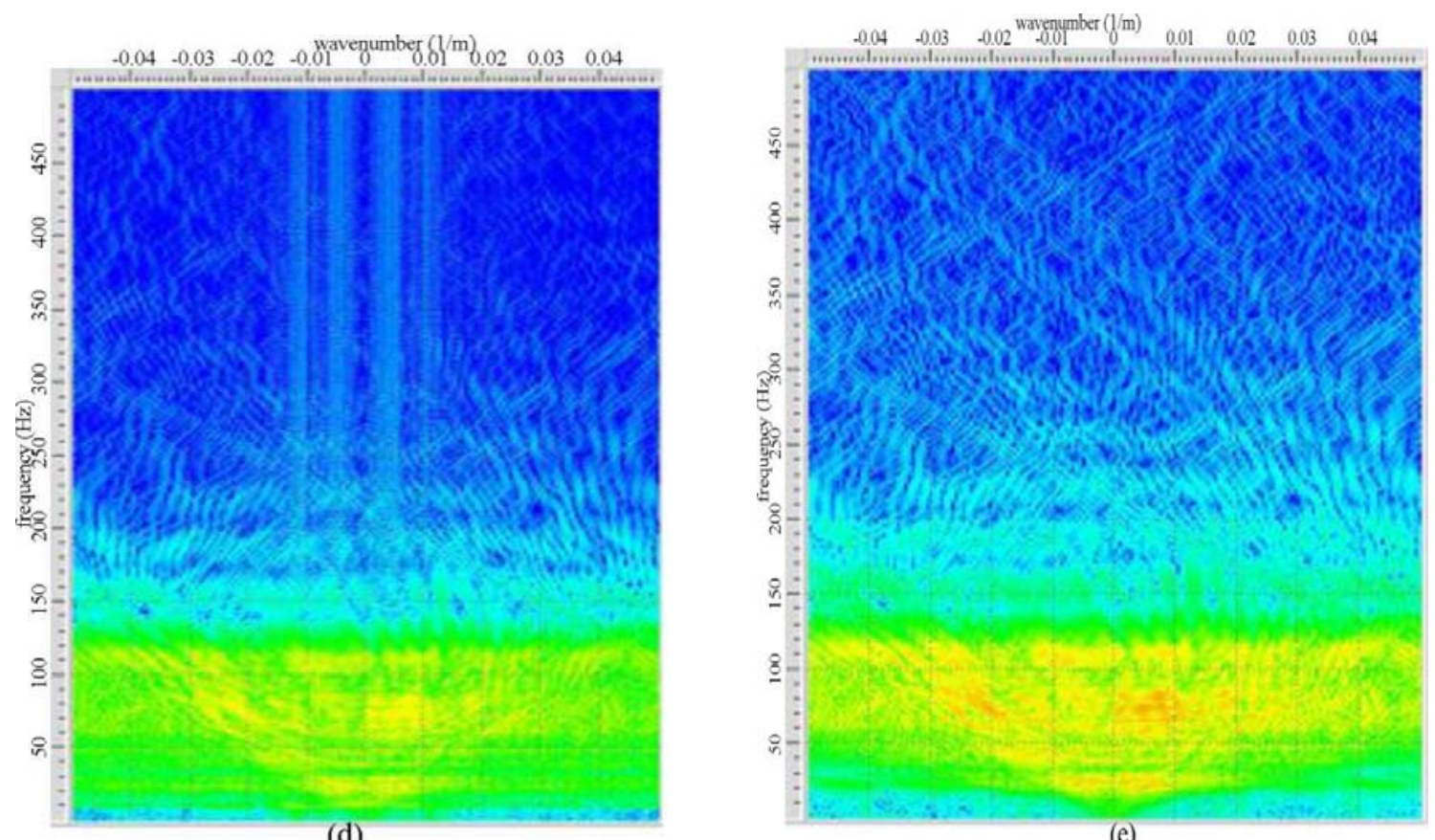

(e)

Fig. 4 (a) was far source point's arrange single shot raw records; (b) was single shot records after 3-D FKK filtering in inline direction; (c) was the filtered noise; (d) was single shot records' frequency spectrum before filtering; (e) was single shot records' frequency spectrum after filtering 

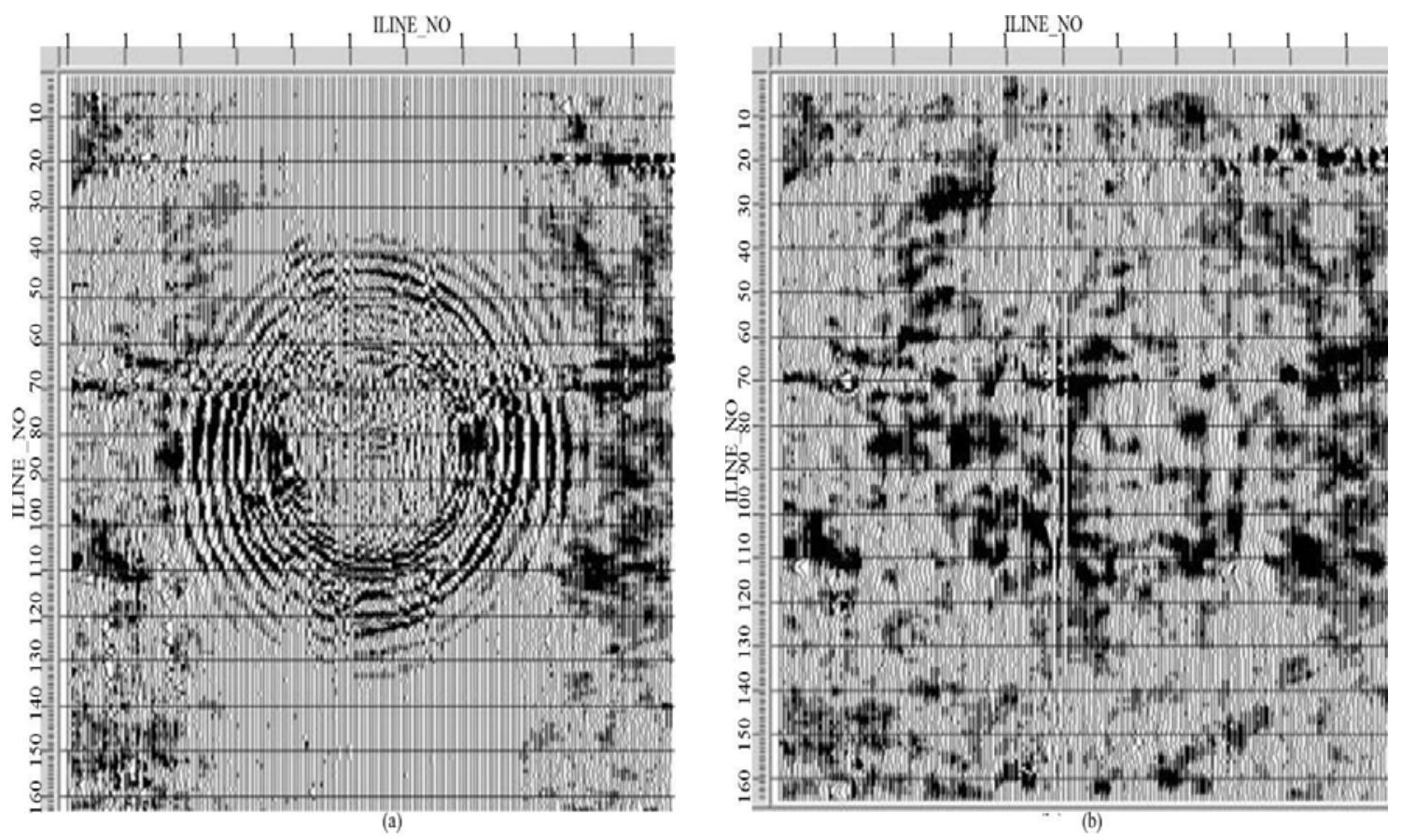

Fig. 5 (a) was a time slice at $1500 \mathrm{~ms}$ of a stack profile before 3-D FKK filtering; (b) was a time slice at 1500 ms of a stack profile after 3-D FKK filtering

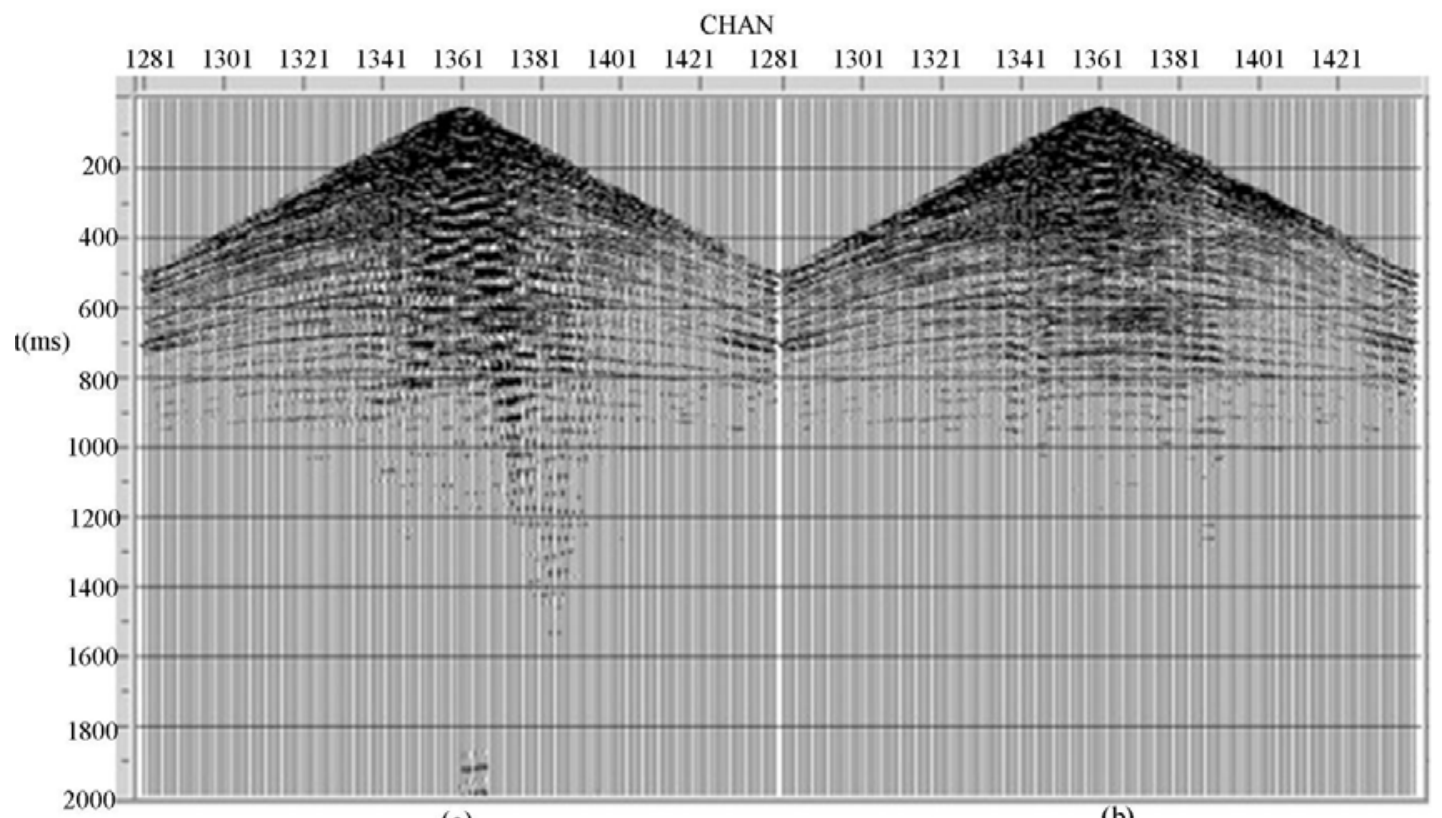

(a)

(b)

Fig. 6 (a) was a typical shot gather after traditional two dimensional FK filtering; (b) was typical shot gather after 3-D FKK filtering in inline direction 